\title{
The Problem of Three Stars: Stability Limit
}

\author{
M. Valtonen ${ }^{1}$, A. Mylläri ${ }^{1}$, V. Orlov ${ }^{2}$ and A. Rubinov ${ }^{2}$ \\ ${ }^{1}$ Department of Physics and Tuorla Observatory, University of Turku, 21500 Piikkiö, Finland \\ email: mavalto@utu.fi \\ ${ }^{2}$ Sobolev Astronomical Institute, St. Petersburg State University, Russia \\ email: vor@astro.spbu.ru
}

\begin{abstract}
The problem of three stars arises in many connections in stellar dynamics: threebody scattering drives the evolution of star clusters, and bound triple systems form long-lasting intermediate structures in them. Here we address the question of stability of triple stars. For a given system the stability is easy to determine by numerical orbit calculation. However, we often have only statistical knowledge of some of the parameters of the system. Then one needs a more general analytical formula. Here we start with the analytical calculation of the single encounter between a binary and a single star by Heggie (1975). Using some of the later developments we get a useful expression for the energy change per encounter as a function of the pericenter distance, masses, and relative inclination of the orbit. Then we assume that the orbital energy evolves by random walk in energy space until the accumulated energy change leads to instability. In this way we arrive at a stability limit in pericenter distance of the outer orbit for different mass combinations, outer orbit eccentricities and inclinations. The result is compared with numerical orbit calculations.
\end{abstract}

Keywords. stellar dynamics, celestial mechanics

\section{Introduction}

Three-body scattering was studied comprehensibly in the pioneering work of Heggie (1975). Among the many results in this work was an expression for energy change when a single body passes by a binary. The calculation was carried out in greater detail by Roy \& Haddow (2003) for a parabolic passage, and by Heggie (2006) for a hyperbolic passage. Valtonen \& Karttunen (2006) calculate the same quantity for a passage in a low-eccentricity elliptic orbit.

In a bound triple system a number of passages takes place one after another. Even though each passage may change the orbits only slightly, a large number of them may lead to an accumulated energy change which eventually leads to the break-up of the triple system. The energy steps in this process may be in one direction only, or they may happen in both directions in the manner of random walk. In either case, the orbits evolve with time toward instability (Valtonen \& Karttunen 2006).

In this paper we study the analytical expressions for the energy change in a single encounter, and simplify them a little for the purpose of the stability study. We then derive an analytical expression for the stability limit which improves the formula given by Valtonen \& Karttunen (2006). This formula also improves the results given in previous works: Golubev (1967), Golubev (1968), Harrington (1977), Eggleton \& Kiseleva (1995), Mardling \& Aarseth (1999), Mardling \& Aarseth (2001) (see Aarseth 2003 and Tokovinin 2004). 


\section{Energy change in a single encounter}

Heggie (1975) and Roy \& Haddow (2003) derive the expression for the relative energy change $\delta \varepsilon / \varepsilon$ in a single parabolic encounter. Heggie (2006) finds the corresponding formula for hyperbolic encounters and shows that it agrees with the result of Roy \& Haddow (2003) at the parabolic limit. Let the binary have masses $m_{1}$ and $m_{2}$, with $M_{12}=m_{1}+m_{2}$, semi-major axis $a_{i}$ and eccentricity $e_{i}$. The third body orbit relative to the barycenter of the binary has pericenter distance $q$, with $Q=q / a_{i}$, and eccentricity $e$. The mass of the third body is $m_{3}$. Its orbital plane relative to the binary plane is described by the usual elements $i, \omega$, and $\Omega$. Then, as was derived in the papers cited above, by the first order perturbation theory

$$
\begin{aligned}
\frac{\delta \varepsilon}{\varepsilon} \simeq & -\frac{\sqrt{\pi}}{4} \frac{m_{3}}{M_{12}} Q^{-3} K^{5 / 2} e^{-(2 / 3) K}\left\{e _ { 1 } \left[\sin \left(2 \omega+n t_{0}\right)(\cos 2 i-1)\right.\right. \\
& -\sin \left(2 \omega+n t_{0}\right) \cos (2 i) \cos (2 \Omega)-3 \sin \left(n t_{0}+2 \omega\right) \cos (2 \Omega) \\
& \left.-4 \sin (2 \Omega) \cos \left(2 \omega+n t_{0}\right) \cos i\right]+e_{2}\left(1-e_{i}^{2}\right)\left[\sin \left(2 \omega+n t_{0}\right)(1-\cos 2 i)\right. \\
& -\sin \left(2 \omega+n t_{0}\right) \cos (2 i) \cos (2 \Omega)-3 \sin \left(n t_{0}+2 \omega\right) \cos (2 \Omega) \\
& \left.-4 \cos \left(n t_{0}+2 \omega\right) \sin (2 \Omega) \cos i\right]+e_{4} \sqrt{1-e_{i}^{2}}\left[-2 \cos (2 i) \cos \left(2 \omega+n t_{0}\right) \sin (2 \Omega)\right. \\
& \left.\left.-6 \cos \left(2 \omega+n t_{0}\right) \sin (2 \Omega)-8 \cos (2 \Omega) \sin \left(2 \omega+n t_{0}\right) \cos i\right]\right\}
\end{aligned}
$$

Here $n$ is the mean motion of the binary and $t_{0}$ is a reference time. The true anomaly of the binary $M=n\left(t-t_{0}\right)$. If we agree that at the pericenter $t=0$, then the value of true anomaly at the pericenter is $M_{0} \equiv 2 \Phi_{0}=-n t_{0}$. The quantity $K$ is defined as

$$
K=\sqrt{2} \sqrt{\frac{M_{12}}{M_{12}+m_{3}}} Q^{3 / 2} .
$$

The functions $e_{1}, e_{2}$ and $e_{4}$ are

$$
\begin{aligned}
& e_{1}=J_{-1}\left(e_{i}\right)-2 e_{i} J_{0}\left(e_{i}\right)+2 e_{i} J_{2}\left(e_{i}\right)-J_{3}\left(e_{i}\right), \\
& e_{2}=J_{-1}\left(e_{i}\right)-J_{3}\left(e_{i}\right), \\
& e_{4}=J_{-1}\left(e_{i}\right)-e_{i} J_{0}\left(e_{i}\right)-e_{1} J_{2}\left(e_{i}\right)+J_{3}\left(e_{i}\right) .
\end{aligned}
$$

Here $J_{-1}, \ldots, J_{3}$ are the Bessel functions.

Let us simplify (2.1) by assuming that $e_{i}$ is small, and use only first order terms. Also we write the dependence on $Q$ with the help of a scale distance $Q_{1}$ :

$$
Q_{1}=2.5\left(1+\frac{m_{3}}{M_{12}}\right)^{1 / 3}
$$

whereby

$$
K=5.59\left(Q / Q_{1}\right)^{3 / 2}
$$

and

$$
K^{5 / 2}=73.9\left(Q / Q_{1}\right)^{15 / 4}
$$

We also approximate the exponential factor by a power-law in the interval $1 \leqslant Q / Q_{1} \leqslant$ 1.5 ; thus

$$
(2.5)^{-3}\left(Q / Q_{1}\right)^{-3} K^{5 / 2} e^{-(2 K / 3)} \simeq 0.11\left(Q / Q_{1}\right)^{-7} .
$$

After all these modifications, the dominant term of (2.1) becomes

$$
\frac{\delta \varepsilon}{\varepsilon} \simeq e_{i} \frac{m_{3}}{M_{12}}\left(\frac{Q}{Q_{1}}\right)^{-7}\left(\frac{1+\cos i}{2}\right)^{2} \sin 2\left(\Phi_{0}-(\omega+\Omega)\right)
$$


Energy change at $\mathrm{m} 3=1, \mathrm{i}=0, \mathrm{Q}=3$

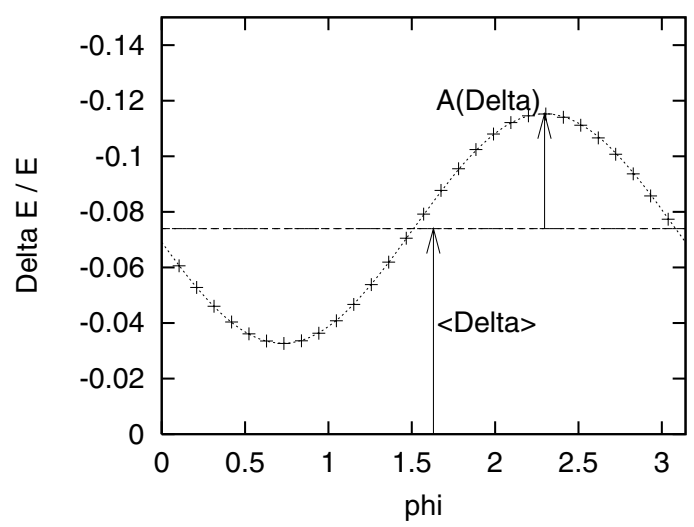

Figure 1. The relative energy change $\delta \varepsilon / \varepsilon$ in a single parabolic encounter between an equal mass circular binary and a single star. The energy change consists of constant shift (marked Delta) and of a sinusoidal variation with amplitude A(Delta).

Thus the energy change is a sinusoidally varying function of the initial phase angle $\left(\Phi_{0}\right)$ of the binary. For initially zero eccentricity binaries, a suitable value to insert in (2.2) is $e_{i} \simeq 0.05$ since the binary eccentricity does not remain zero during the encounter but changes to a non-zero value.

Valtonen \& Karttunen (2006) derive a similar result by using a low-eccentricity outer orbit instead of a parabolic orbit. They find approximately for $e_{i}=0$ that

$$
\frac{\delta \varepsilon}{\varepsilon} \simeq 0.03 \frac{m_{3}}{M_{12}}\left(\frac{Q}{Q_{1}}\right)^{-7}\left(\frac{1+\cos i}{2}\right)^{2} \cos 2\left(\Phi_{0}-\Omega\right) .
$$

Because of zero eccentricity, we have put $\omega=0$, since the orientation of the major axis is of no consequence.

Numerical calculations show that the inclination function is in fact somewhat more complicated than in (2.2) and (2.3). We will find below an expression which is more suitable for the stability study. Note that the

$$
\left(\frac{1+\cos i}{2}\right)^{2}
$$

function cannot possibly be correct since it would make the energy change at retrograde orbit zero.

The functional forms of the relative energy change have been tested by single encounter parabolic orbit calculations. Fig. 1 shows that the $\delta \varepsilon / \varepsilon$ variation is sinusoidal. Fig. 2 shows the amplitude of the sinusoidal variation as a function of $Q$. It may be modelled as pure power-law at small $Q$, and by a combination of power-law and exponential at larger $Q$ (dotted line). This simple sinusoidal behavior is valid only if $Q \geqslant Q_{s t}$ where $Q_{s t}$ may be called the stability limit of a single encounter. Fig. 3 shows that $Q_{s t}$ depends strongly on the inclination. The stability limit also depends on the mass of the third body, as shown by Fig. 4. The limit may be defined in two ways: either from the $Q$-distance where exchanges between binary bodies and third bodies first start, or from the innermost point to which a power-law dependence on $Q$ is valid. These stability limits are compared 


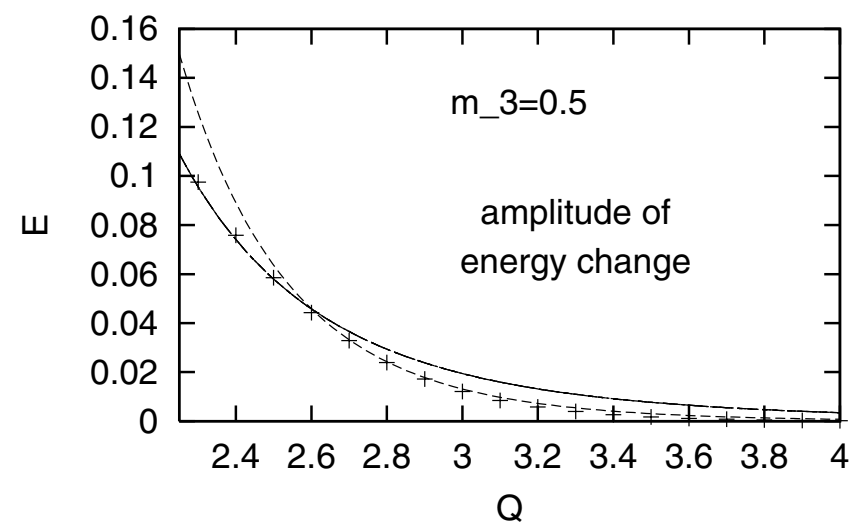

Figure 2. The amplitude of the sinusoidal (relative) energy change in a parabolic single star binary encounter, as a function of the pericenter distance $Q$ (in units of the binary semi-major axis). A pure power-law (solid line) fits at low $Q$ while at higher $Q$ a power-law times an exponential gives a better fit.

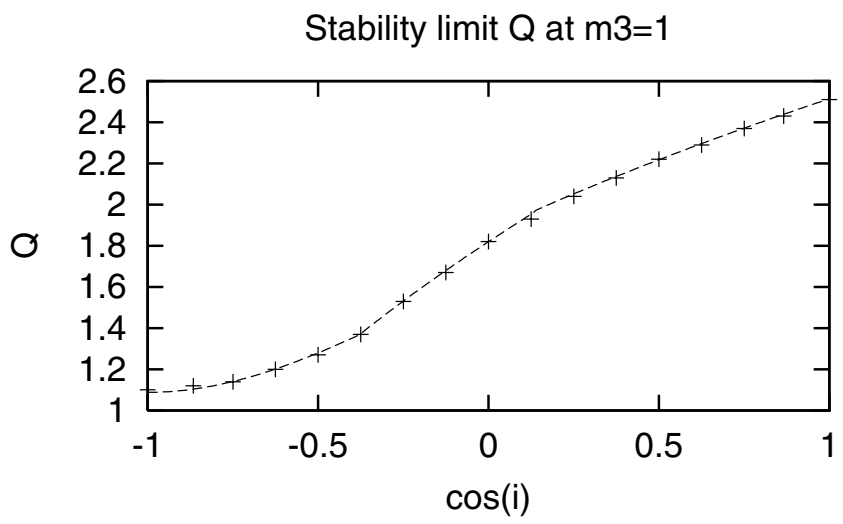

Figure 3. The stability limit $Q_{s t}$ as a function of $\cos i$ in a parabolic single - star - binary encounter.

with the expression

$$
2.1\left(1+\frac{m_{3}}{M_{12}}\right)^{1 / 3}
$$

by a dashed line in Fig. 4 . Note the connection with the scale distance $Q_{1}$ defined above.

The dependence of $\delta \varepsilon / \varepsilon$ on $Q$ and $m_{3} / M_{12}$ is shown in Fig. 5, while the dependence on $Q$ and $i$ is displayed in Fig. 6. The lines show that simple analytical models describe the data when $Q>Q_{s t}$.

\section{Evolution of the outer orbit}

The energy of the outer orbit $E_{\text {out }}$ is connected to the binary energy $E_{B}$ by

$$
\frac{E_{B}}{E_{\text {out }}}=\frac{m_{1} m_{2}}{M_{12} m_{3}} \frac{a}{a_{i}}=\frac{m_{1} m_{2}}{M_{12} m_{3}} \frac{Q}{1-e}
$$




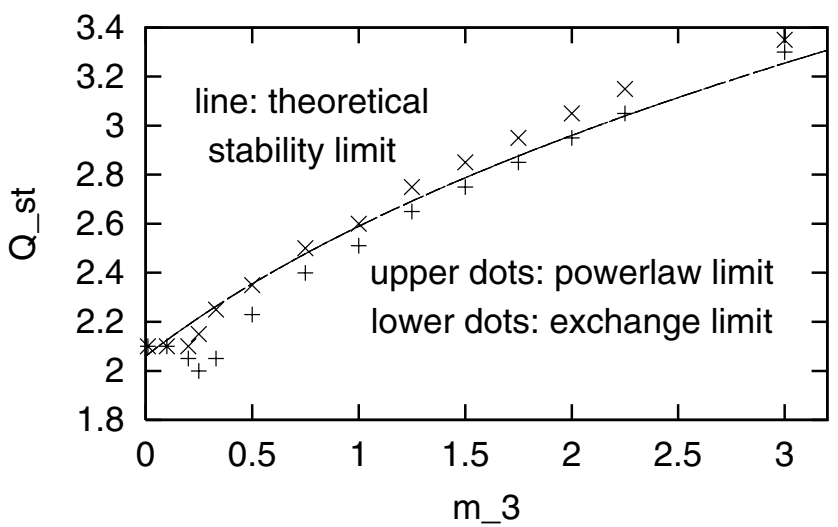

Figure 4. The stability limit $Q_{s t}$ as a function of third body mass, $m_{3} / M_{12}$, for $i=0^{\circ}$. The points may be defined either by the lower limit of power-law description in $\delta \varepsilon / \varepsilon \alpha Q^{-m}$, where $m \simeq 7$, or by the limit where exchanges begin between the binary members and the third body.

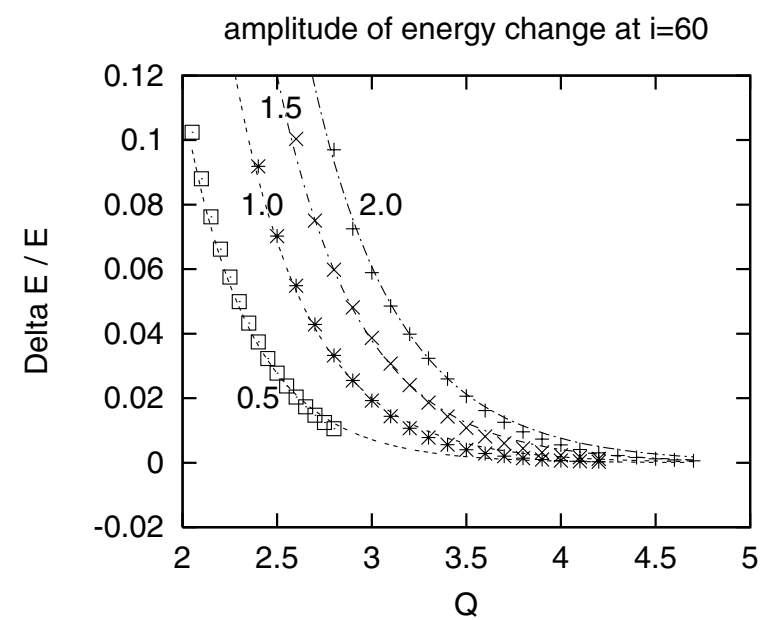

Figure 5. The dependence of $\delta \varepsilon / \varepsilon$ on pericentric distance $Q$ at various mass values $m_{3} / M_{12}$.

where $a$ is the semi-major axis of the outer orbit. If $m_{1}=m_{2}$,

$$
\frac{E_{B}}{E_{\text {out }}} \simeq \frac{M_{12}}{m_{3}} \frac{Q / Q_{1}}{1-e} .
$$

Therefore the relative change in the binary energy

$$
\frac{\triangle E_{B}}{E_{B}}=-\frac{\triangle E_{\text {out }}}{E_{\text {out }}} \frac{E_{\text {out }}}{E_{B}} \simeq-\frac{(1-e)}{Q / Q_{1}} \frac{m_{3}}{M_{12}} \frac{\triangle E_{\text {out }}}{E_{\text {out }}} .
$$

Let us assume that the triple system breaks up when $\triangle E_{\text {out }} \simeq E_{\text {out }}$. It can happen after $N$ steps of random walk. If the step size $\triangle E_{\text {out }} / E_{\text {out }}=x$, then

$$
\sqrt{N} x=1
$$

or $N=x^{-2}$. 


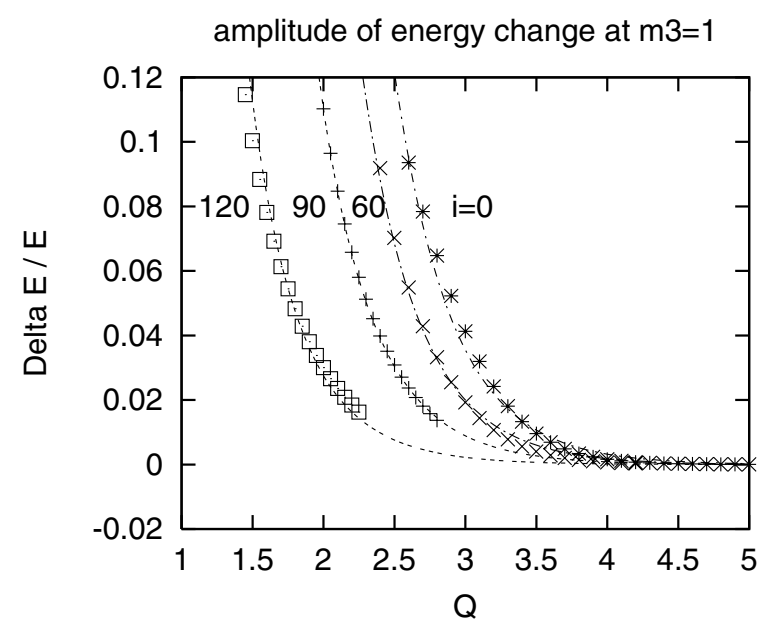

Figure 6. The dependence of $\delta \varepsilon / \varepsilon$ on pericentric distance $Q$ at various inclination values.

If $x=10^{-2}, N=10^{4}$. In other words, if we put

$$
\frac{\triangle E_{\text {out }}}{E_{\text {out }}}=10^{-2} \text {, }
$$

the system should be stable over $10^{4}$ revolutions of the outer orbit. Thus

$$
\frac{\triangle E_{\text {out }}}{E_{\text {out }}} \simeq 10^{-2} \frac{(1-e)}{Q / Q_{1}} \frac{m_{3}}{M_{12}} .
$$

Let us put this equal to the amplitude of $\delta \varepsilon / \varepsilon$ :

$$
10^{-2} \frac{(1-e)}{Q / Q_{1}} \frac{m_{3}}{M_{12}}=0.03 \frac{m_{3}}{M_{12}}\left(\frac{Q}{Q_{1}}\right)^{-7}\left(\frac{1+\cos i}{2}\right)^{2} .
$$

Solve for $Q$ :

$$
Q \simeq 3\left(1+\frac{m_{3}}{M_{12}}\right)^{1 / 3}(1-e)^{-1 / 6}\left(\frac{1+\cos i}{2}\right)^{1 / 3} .
$$

This value is referred to as the stability limit $Q_{s t}$.

\section{Stability experiments}

Numerical orbit calculations have been carried for triple systems where the binary members are of equal mass. The value of $e$ is varied in 5 steps from 0 to 0.9 and $m_{3} / M_{12}$ in the range from 0.0005 to 5 . Inclination values from zero to $180^{\circ}$ have been studied. For the numerical integration of the equations of motion we used the code kindly provided by S. Mikkola that applies Wisdom-Holman method with time transformation in the extended phase-space (for the details see Mikkola 1997). The system is classified as stable if after $N=10^{4}$ revolutions of the outer orbit there have been no escapes and no exchanges of binary members. The stability limit for fixed $e, m_{3} / M_{12}$ and $i$ is determined such that the triple systems are stable down to this value of $Q$ for any value of $\omega$ and $\Omega$. There may be stable systems even at smaller $Q$, but there is no guarantee that for $Q<Q_{s t}$ the system is definitely stable. 

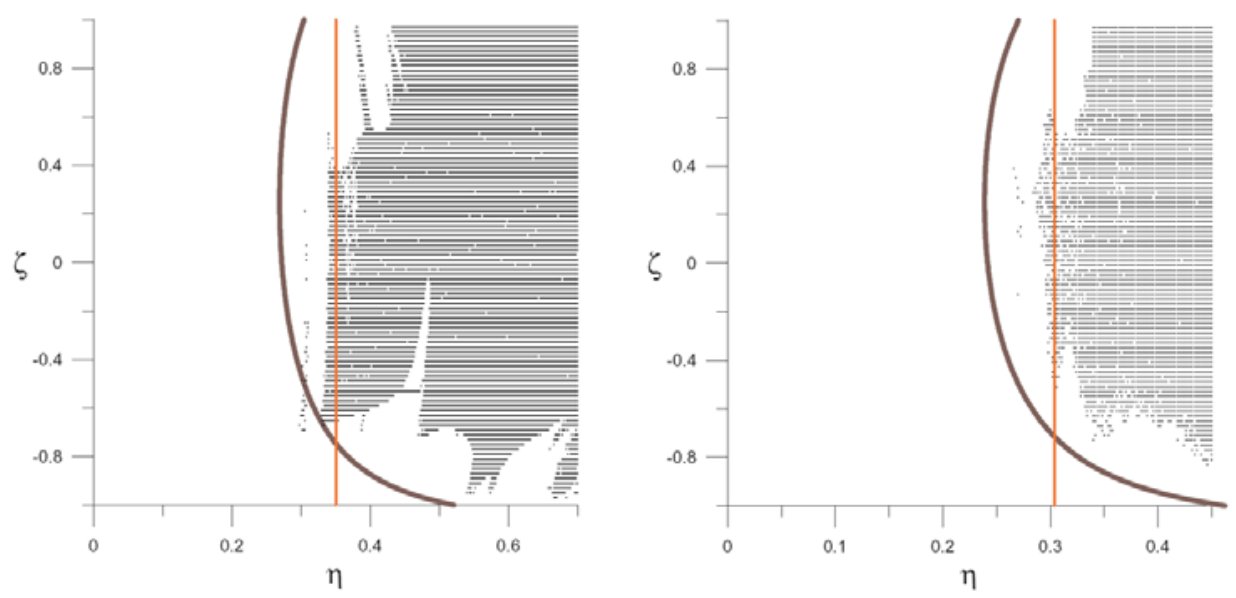

Figure 7. Results from numerical stability calculations for hierarchical triple systems for up to $10^{4}$ revolutions of the outer orbit. The parameters are $\eta=a_{i} / a$ and $\xi=\cos i$. Every dot represents an unstable orbit. The vertical line follows the Mardling \& Aarseth (2001) criterion while the curved line is from this work. There should be no dots to the left of the line if the boundary is correct. The case of $e=0, m_{3}=0.1$ (left panel), $m_{3}=1$ (right panel) and $m_{1}=m_{2}=1$.
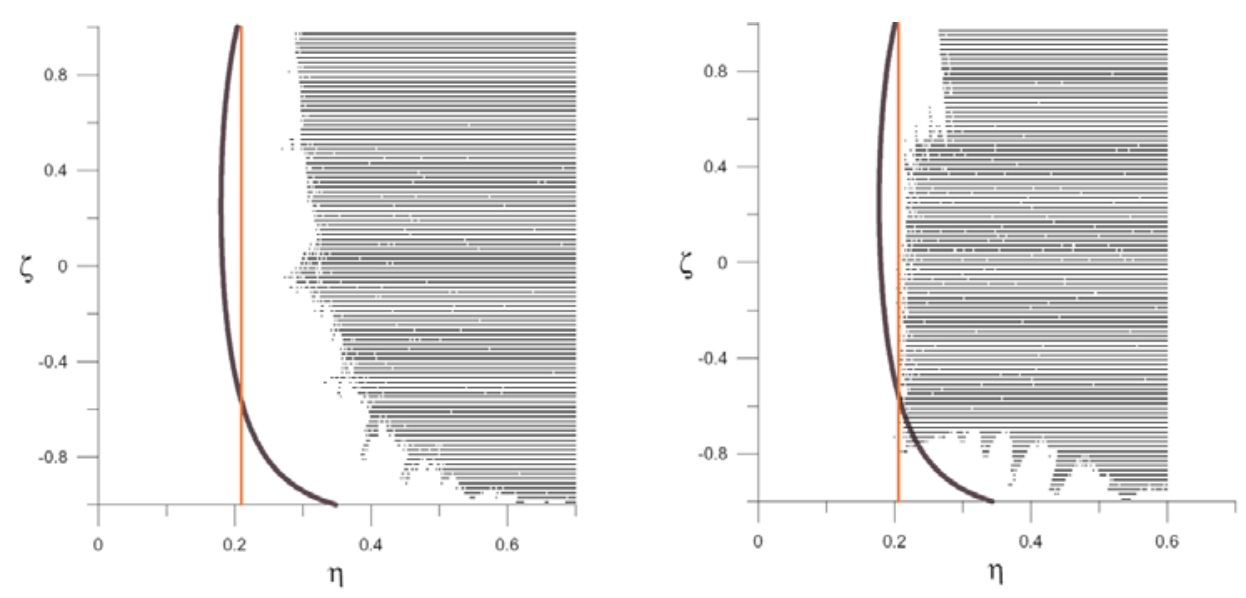

Figure 8. Same as Fig. 7, except that $e=0.3, m_{3}=0.01$ (left), $m_{3}=0.1$ (right).

As we mentioned earlier, the functional forms present in (3.1) are not necessarily optimal for this problem. Therefore small variants have been tried. For the inclination, the functional form

$$
\left(\frac{7}{4}+\frac{1}{2} \cos i-\cos ^{2} i\right)^{1 / 3}
$$

have been found to improve the fit relative to the $[(1+\cos i) / 2]^{1 / 3}$ form. Then the whole formula becomes

$$
Q_{s t}=3\left(1+m_{3} / M_{12}\right)^{1 / 3}(1-e)^{-1 / 6}\left(\frac{7}{4}+\frac{1}{2} \cos i-\cos ^{2} i\right)^{1 / 3} .
$$

The agreement between experiments and theory is shown in Figs. 7-11. The expression is not good for $0.025<m_{3} / M_{12}<0.17$ for retrograde orbits. In such cases one may use 

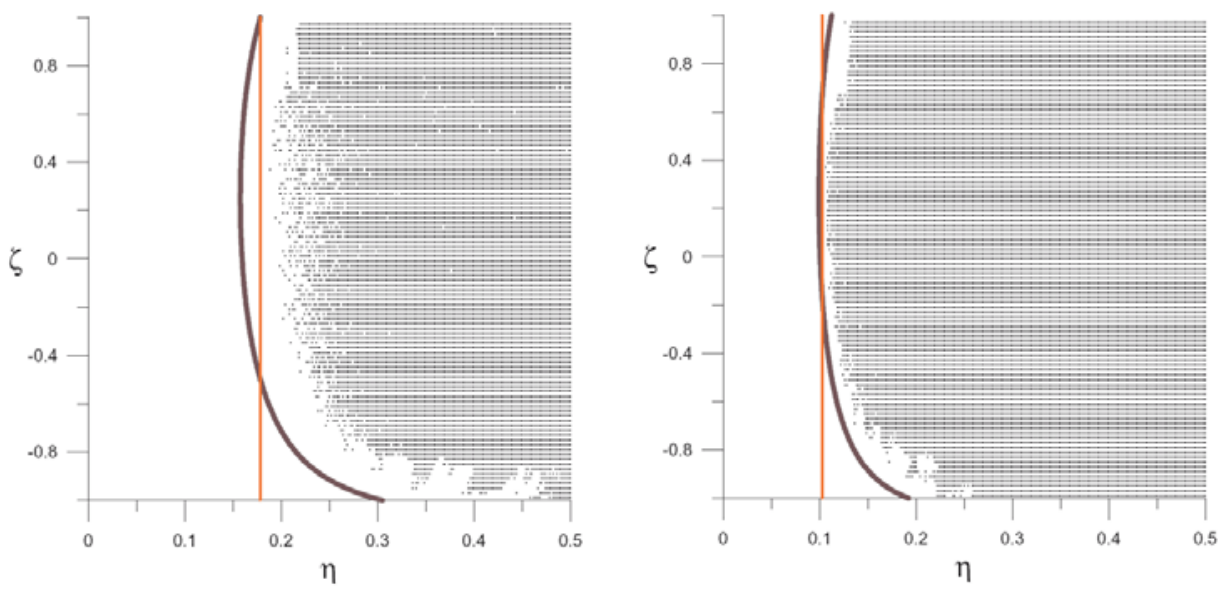

Figure 9. Same as Fig. 7, except that $e=0.3, m_{3}=1$ (left), $m_{3}=10$ (right).
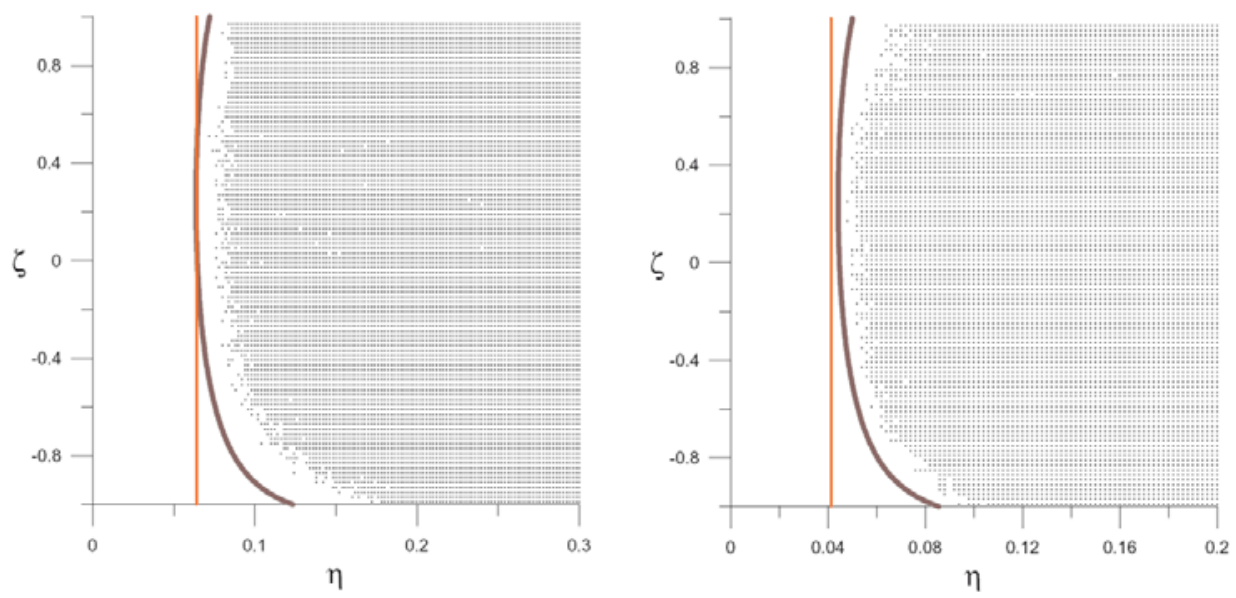

Figure 10. Same as Fig. 7, except that $e=0.7, m_{3}=0.333$ (left), $m_{3}=5$ (right).
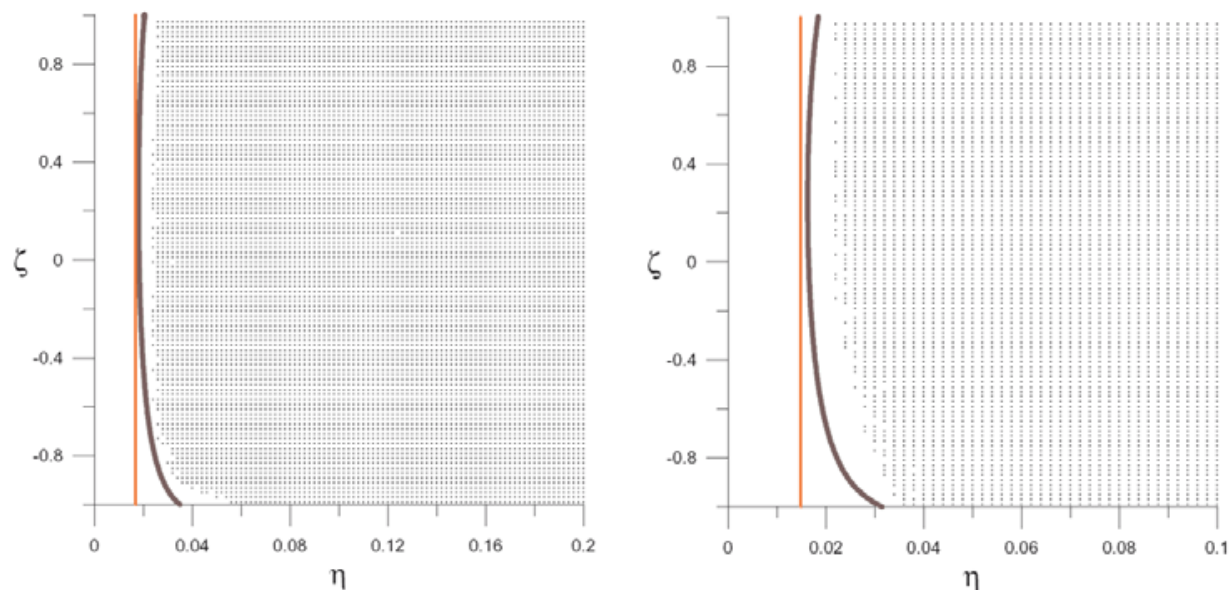

Figure 11. Same as Fig. 7, except that $e=0.9, m_{3}=0.2$ (left), $m_{3}=1$ (right). 
$\cos i=0$ in the formula whenever $\cos i<0$. Outside this mass range the formula works well, at least for $0<m_{3} / M_{12} \leqslant 0.025$ and for $0.17 \leqslant m_{3} / m_{B} \leqslant 5$, where we have been able to test it. Typically there is about $5 \%$ safely margin in $Q_{s t}$, i.e. the limit could be reduced by this much and only very few exceptions to the rule would arise.

\section{Conclusions}

As we mentioned in the introduction, several stability criteria have been proposed. In general, they are simpler formulae than what is presented in this paper. The Valtonen \& Karttunen (2006) formula is an earlier and less accurate formulation of the same theory as is presented here. We have compared our data with Mardling \& Aarseth (2001) criteria. In general it is good, but as can be seen in Fig. 7 it sometimes doesn't work in the middle inclinations. Zhuchkov, Orlov \& Rubinov (2006) have shown that the stability criteria of Golubev, Harrington and Tokovinin are not always good. It would be an interesting project to compare the other criteria with ours and with numerical orbit calculations, but it will be left to another paper.

\section{References}

Aarseth, S. 2003, Gravitational N-Body Simulations Cambridge Univ. Press, Cambridge, p. 151. Eggleton, P. \& Kiseleva, L. 1995, ApJ 455, 640

Golubev, V. G. 1967, Sov. Phys. Dokl. 12, 529

Golubev, V. G. 1968, Sov. Phys. Dokl. 13, 373

Harrington, R. S. 1977, Rev. Mexicana AyA 3, 139

Heggie, D. C. 1975 , MNRAS 173, 729

Heggie, D. C. 2006, in C. F. Flynn (ed.), Few Body Problem: Theory and Computer Simulations, Ann. Univ. Turku, Ser. 1 A, Vol. 358, p. 20

Mardling, R. \& Aarseth, S. 1999, in B. A. Steves \& A. E. Roy (ed.), The Dynamics of Small Bodies in the Solar System Studies, Kluwer, p. 385

Mardling, R. \& Aarseth, S. 2001, MNRAS 321398

Mikkola, S. 1997, Cel. Mech. Dyn. Astr., 67, 145

Roy, A. \& Haddow, M. 2003, Cel. Mech. Dyn. Astr., 87, 411

Tokovinin, A. 2004, Rev. Mexicana AyA 21, 7

Valtonen, M. \& Karttunen, H. 2006, The Three-Body Problem, Cambridge U. Press, Cambridge, Chapter 2

Zhuchkov, R., Orlov, V., \& Rubinov, A. 2006, in C. F. Flynn (ed.), Few Body Problem: Theory and Computer Simulations, Ann. Univ. Turku, Ser. 1 A, Vol. 358, p. 79 